\title{
Imaging inflammation in atherosclerosis: Exploring all avenues
}

\author{
Ahmed Haider, PhD, ${ }^{a, b}$ Susan Bengs, PhD, ${ }^{a, b}$ and Catherine Gebhard, MD, \\ $\mathrm{PhD}^{\mathrm{a}, \mathrm{b}}$ \\ a Department of Nuclear Medicine, University Hospital Zurich, Zurich, Switzerland \\ b Center for Molecular Cardiology, University of Zurich, Schlieren, Switzerland
}

Received Mar 8, 2020; accepted Mar 9, 2020

doi: $10.1007 / \mathrm{s} 12350-020-02103-6$

See related article, pp. 2506-2513

Despite remarkable advances in preventive and therapeutic strategies, cardiovascular disease (CVD) remains the primary source of death and disability worldwide. ${ }^{1}$ Further, the demographic shift towards an older population will result in increasing numbers of patients, among whom heart disease is the leading cause of death. In view of this global burden, personalized risk-stratification tools and a cost-effective management of CVD are urgently needed. Although rapid innovations in atherosclerosis imaging have widened its applications from anatomical plaque detection to physiological assessment and complex evaluation of vascular biology, non-invasive imaging tools that flag the 'vulnerable' patient are still lacking. Indeed, although the presence of coronary artery stenosis is associated with subsequent events, most myocardial infarctions occur in segments with non-obstructive rather than obstructive disease. ${ }^{2}$ As recent landmark trials have further highlighted the fundamental role of inflammation during all stages of

Funding CG was supported by grants from the Swiss National Science Foundation (SNSF), the Olga Mayenfisch Foundation, Switzerland, the OPO Foundation, Switzerland, the Novartis Foundation, Switzerland, the Swiss Heart Foundation, the Helmut Horten Foundation, Switzerland, the EMDO Foundation, Switzerland, and the Iten-Kohaut Foundation, Switzerland. SB and AH were supported by the University of Zurich (UZH) Foundation. SB was supported by the Swiss Heart Foundation, Switzerland.

See related article, https://doi.org/10.1007/s12350-020-02046-y

Reprint requests: Catherine Gebhard, MD, PhD, Department of Nuclear Medicine, University Hospital Zurich, Raemistrasse 100, 8091 Zurich, Switzerland; Catherine.Gebhard@usz.ch

J Nucl Cardiol 2021;28:2514-7.

$1071-3581 / \$ 34.00$

Copyright (c) 2020 American Society of Nuclear Cardiology. atherosclerosis, the assessment of 'residual inflammatory risk' has been proposed as a promising strategy for improving CVD risk prediction and providing guidance for novel precision treatments. ${ }^{3,4}$

Several molecular imaging modalities including ${ }^{99 m}$ Tc-based single-photon emission computed tomography (SPECT) and ${ }^{18} \mathrm{~F}$-fluorodeoxyglucose $\left({ }^{18} \mathrm{~F}\right.$-FDG) positron emission tomography (PET) have been developed for the non-invasive detection of vascular inflammatory activities ${ }^{5,6}$ (Figure 1). ${ }^{18}$ F-FDG-PET has been used to detect macrophage-rich atherosclerotic lesions and as a surrogate endpoint for vascular interventional drug trials. ${ }^{7}$ However, it is limited by its moderate reproducibility and sensitivity for the detection of coronary atherosclerotic changes. In addition, ${ }^{18}$ F-FDG-PET has logistical and technical constraints including the need to suppress myocardial and peripheral muscle FDG uptake for the analysis as well as the high FDG uptake in structures close to the vessel wall. Hence, given the small size of the atherosclerotic lesion and the continuous cardiac and respiratory movements during PET acquisition, nuclear imaging of atherosclerosis remains challenging. Therefore, high-sensitivity PET and SPECT imaging probes that specifically target inflammatory cells in the vessel wall would greatly facilitate the non-invasive evaluation of the vulnerable plaque.

In this issue of the Journal of Nuclear Cardiology, Meester et al. ${ }^{8}$ analyzed the diagnostic potential of DOTA-JR11, labeled with ${ }^{111}$ indium $\left({ }^{111} \mathrm{In}\right)$, in a mouse model of atherosclerosis as well as in human carotid endarterectomy tissue. ${ }^{111}$ In-DOTA-JR 11 targets the upregulated somatostatin receptor subtype $2\left(\mathrm{SST}_{2}\right)$ on activated macrophages and might thus provide functional information about plaque activity and stability. The authors report that aortic plaques were detectable in all atherosclerotic mice, providing a proof-of-concept for the utility of ${ }^{111}$ In-DOTA-JR11 in preclinical 

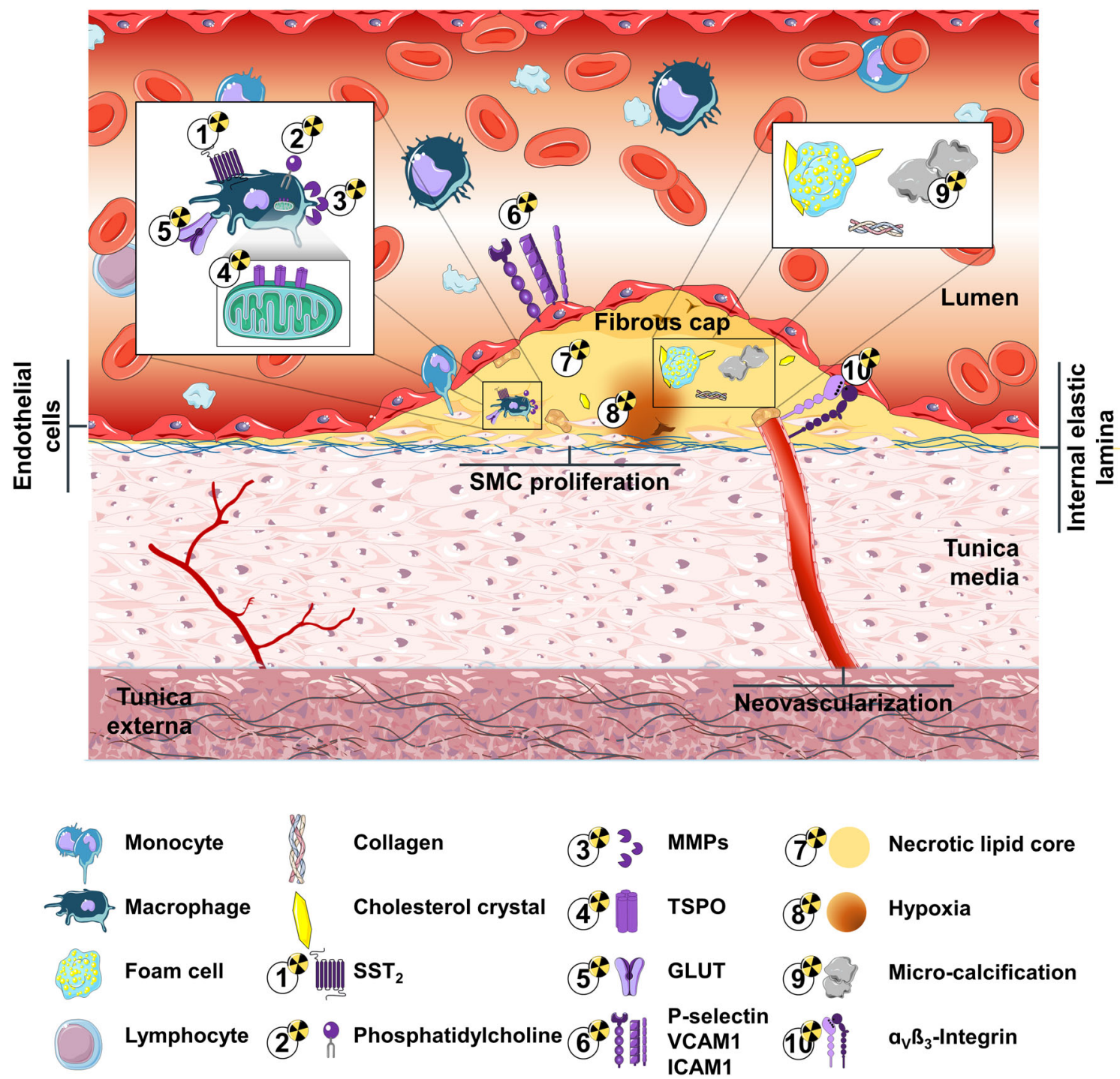

Figure 1. Molecular targets and selected probes for atherosclerosis imaging by positron emission tomography (PET) and single-photon emission computed tomography (SPECT). (1) ${ }^{68}$ Ga-DOTA(Tyr3)-octreotate $\left({ }^{68} \mathrm{Ga}\right.$-DOTATATE), ${ }^{111} \mathrm{In}$-DOTA-JR11. (2) ${ }^{11} \mathrm{C}$-choline. (3) ${ }^{99 \mathrm{~m}} \mathrm{Tc}-\mathrm{RP} 805,{ }^{111} \mathrm{In}$ RP702. (4) ${ }^{11} \mathrm{C}-\mathrm{PK} 11195,{ }^{18} \mathrm{~F}-\mathrm{GE} 180$. (5) ${ }^{18} \mathrm{~F}$-fluorodeoxyglucose $\left({ }^{18} \mathrm{~F}-\mathrm{FDG}\right)$. (6) ${ }^{99 \mathrm{~m}} \mathrm{Tc}-$ cAbVCAM1-5. (7) ${ }^{124}$ I-hypericin. (8) ${ }^{18} \mathrm{~F}$-fluoromisonidazole $\left({ }^{18} \mathrm{~F}\right.$-FMISO). (9) ${ }^{18} \mathrm{~F}$-sodium fluoride $\left({ }^{18} \mathrm{~F}-\mathrm{NaF}\right)$. (10) ${ }^{18} \mathrm{~F}-$ Galacto-RGD, ${ }^{68} \mathrm{Ga}-\mathrm{NOTA}-\mathrm{RGD}$. GLUT, glucose transporter; $M M P s$, matrix metalloproteinases; $S S T_{2}$ somatostatin receptor 2; TSPO, translocator protein.

atherosclerosis imaging. Further, ${ }^{111}$ In-DOTA-JR11 colocalized with CD68- and $\mathrm{SST}_{2}$-expressing cells on human carotid tissue sections indicating that the tracer is suitable to detect inflammatory activities in human atherosclerotic plaques. Their data are consistent with previous reports unveiling a close relationship between CVD risk factors, coronary calcium burden, and arterial uptake of ${ }^{68} \mathrm{Ga}$-DOTA-(Tyr $\left.{ }^{3}\right)$-octreotate $\left({ }^{68} \mathrm{Ga}\right.$-DOTATATE) - the most extensively validated $\mathrm{SST}_{2}$-targeted tracer. ${ }^{9}$ Indeed, in a prospective clinical investigation, Tarkin et al. recently demonstrated that ${ }^{68} \mathrm{Ga}$ -
DOTATATE can be used to differentiate between highrisk vs. low-risk carotid and coronary atherosclerotic lesions. ${ }^{10}$ Further, ${ }^{68} \mathrm{Ga}$-DOTATATE was superior to ${ }^{18}$ F-FDG-PET in discriminating between high-risk vs. low-risk human atherosclerotic plaques, underlining the capability of $\mathrm{SST}_{2}$-targeted tracers in capturing vulnerable plaque features. ${ }^{11}$

In contrast to DOTATATE, DOTA-JR11 belongs to the new generation of $\mathrm{SST}_{2}$ ligands that are based on antagonistic structures and exhibit more favorable receptor binding, pharmacokinetics, and biodistribution 
properties. ${ }^{12}$ It should be noted, however, that $\mathrm{SST}_{2^{-}}$ directed probes have originally been developed and approved for the detection of $\mathrm{SST}_{2}$ overexpression on neuroendocrine tumors. $^{13}$ As such, DOTA-JR11 has recently shown promise in exhibiting a several-fold higher tumor uptake than DOTATATE, ${ }^{14}$ thereby highlighting its potential for a broad clinical applicability. Meester et al. now extend previous findings by demonstrating that DOTA-JR11, when labeled with indium-111, is sensitive to atherosclerotic plaque detection. Although this is an important finding, the authors did not perform a direct comparison between ${ }^{111}$ In-DOTA-JR11 SPECT and ${ }^{68} \mathrm{Ga}$-DOTATATE PET. Thus, whether second-generation $\mathrm{SST}_{2}$-based probes will outperform ${ }^{68} \mathrm{Ga}$-DOTATATE in atherosclerosis imaging will have to be tested in future studies. Further, $\mathrm{N}$-terminal radiometal modifications may substantially affect the performance characteristics of DOTA-JR $11 .{ }^{15}$ Given that gallium-68 labeled DOTA-JR11 $\left({ }^{68} \mathrm{Ga}-\right.$ DOTA-JR11) has not yet been evaluated for the imaging of coronary vascular inflammation, it is unclear how radiometal exchange between ${ }^{111} \mathrm{In}$ and ${ }^{68} \mathrm{Ga}$ will affect tracer performance. Nonetheless, a recent first-in-man study with ${ }^{68}$ Ga-DOTA-JR11 demonstrated pharmacokinetics and dosimetric data that compared favorably with ${ }^{68} \mathrm{Ga}$-DOTATATE, ${ }^{16}$ thus, paving the way for a head-to-head comparison between ${ }^{68}$ Ga-DOTA-JR11 and ${ }^{68} \mathrm{Ga}$-DOTATATE PET in atherosclerosis imaging.

Meester et al. conclude their discussion by stressing the need for future trials demonstrating the clinical utility of DOTA-JR11-based probes in plaque phenotyping. Indeed, provided that an increase in DOTA-JR11 signal is associated with the progression of atherosclerosis and the risk of cardiovascular events, high specific uptake of DOTA-JR11 might not only identify patients with high-risk plaques, but also individuals who could benefit from novel anti-inflammatory treatments. However, pertaining to a wider clinical use of $\mathrm{SST}_{2}$ imaging, it will be indispensable to consider the influence of sex on the complexity of atherosclerotic plaque morphology and activity. The study by Meester et al. did not address this important issue; nonetheless, sexual dimorphism in the association between inflammation and coronary artery disease has recently been demonstrated. ${ }^{17,18}$ Further, recent advances in coronary computed tomography angiography (CCTA) technology allow to detect coronary inflammation by capturing perivascular fat attenuation. ${ }^{19}$ Given the widespread use of CCTA as well as novel CCTA acquisition techniques ranging in a sub-millisievert fraction of effective radiation dose, ${ }^{20}$ the prognostic performance of $\mathrm{SST}_{2}$-targeted probes will have to be validated against novel imaging biomarkers derived from CCTA before they may advance from research tools to clinical applications. Future studies should also address potential synergistic applications of nuclear imaging techniques and CCTA, with the ultimate goal to identify the vulnerable plaque, the vulnerable artery, and, most importantly, the vulnerable patient.

\section{Disclosure}

All authors have the following to disclose: The University Hospital of Zurich holds a research contract with GE Healthcare. CG has received research grants from the Novartis Foundation, Switzerland.

\section{References}

1. Roth GA, Abate D, Abate KH, Abay SM, Abbafati C, Abbasi N, et al. Global, regional, and national age-sex-specific mortality for 282 causes of death in 195 countries and territories, 1980-2017: A systematic analysis for the Global Burden of Disease Study 2017. The Lancet 2018;392:1736-88.

2. Chang HJ, Lin FY, Lee SE, Andreini D, Bax J, Cademartiri F, et al. Coronary atherosclerotic precursors of acute coronary syndromes. J Am Coll Cardiol 2018;71:2511-22.

3. Tardif JC, Kouz S, Waters DD, Bertrand OF, Diaz R, Maggioni $\mathrm{AP}$, et al. Efficacy and safety of low-dose colchicine after myocardial infarction. N Engl J Med 2019;381:2497-505.

4. Ridker PM, Everett BM, Thuren T, MacFadyen JG, Chang WH, Ballantyne C, et al. Antiinflammatory therapy with canakinumab for atherosclerotic disease. N Engl J Med 2017;377:1119-31.

5. Tarkin JM, Dweck MR, Evans NR, Takx RAP, Brown AJ, Tawakol A, et al. Imaging atherosclerosis. Circ Res 2016;118:750-69.

6. Meester EJ, Krenning BJ, de Swart J, Segbers M, Barrett HE, Bernsen MR, et al. Perspectives on small animal radionuclide imaging; considerations and advances in atherosclerosis. Front Med (Lausanne) 2019;6:39.

7. Ogawa M, Magata Y, Kato T, Hatano K, Ishino S, Mukai T, et al. Application of 18F-FDG PET for monitoring the therapeutic effect of antiinflammatory drugs on stabilization of vulnerable atherosclerotic plaques. J Nucl Med 2006;47:1845-50.

8. Meester EJ, Krenning BJ, de Blois E, de Jong M, van der Steen $\mathrm{AFW}$, Bernsen MR, et al. Imaging inflammation in atherosclerotic plaques, targeting SST2 with [111In]In-DOTA-JR11 2020.

9. Rominger A, Saam T, Vogl E, Ubleis C, la Fougere C, Forster S, et al. In vivo imaging of macrophage activity in the coronary arteries using 68Ga-DOTATATE PET/CT: Correlation with coronary calcium burden and risk factors. J Nucl Med 2010;51:193-7.

10. Tarkin JM, Joshi FR, Evans NR, Groves AM, Gopalan D, Manavaki $\mathrm{R}$, et al. Use of somatostatin receptor PET to differentiate between high-risk and low-risk atherosclerotic lesions: A prospective clinical study. The Lancet 2016;387:S97.

11. Tarkin JM, Joshi FR, Evans NR, Chowdhury MM, Figg NL, Shah $\mathrm{AV}$, et al. Detection of atherosclerotic inflammation by (68)GaDOTATATE PET compared to [(18)F]FDG PET imaging. J Am Coll Cardiol 2017;69:1774-91.

12. Fani M, Nicolas GP, Wild D. Somatostatin receptor antagonists for imaging and therapy. J Nucl Med 2017;58:61s-6s.

13. Barrio M, Czernin J, Fanti S, Ambrosini V, Binse I, Du L, et al. The impact of somatostatin receptor-directed PET/CT on the 
management of patients with neuroendocrine tumor: A systematic review and meta-analysis. J Nucl Med 2017;58:756-61.

14. Wild D, Fani M, Fischer R, Del Pozzo L, Kaul F, Krebs S, et al. Comparison of somatostatin receptor agonist and antagonist for peptide receptor radionuclide therapy: A pilot study. J Nucl Med 2014;55:1248-52.

15. Fani M, Braun F, Waser B, Beetschen K, Cescato R, Erchegyi J, et al. Unexpected sensitivity of sst2 antagonists to N-terminal radiometal modifications. J Nucl Med 2012;53:1481-9.

16. Krebs S, Pandit-Taskar N, Reidy D, Beattie BJ, Lyashchenko SK, Lewis JS, et al. Biodistribution and radiation dose estimates for 68Ga-DOTA-JR11 in patients with metastatic neuroendocrine tumors. Eur J Nucl Med Mol Imaging 2019;46:677-85.

17. Fiechter M, Haider A, Bengs S, Maredziak M, Burger IA, Roggo A, et al. Sex differences in the association between inflammation and ischemic heart disease. Thromb Haemost 2019;119:1471-80.
18. Fiechter M, Haider A, Bengs S, Maredziak M, Burger IA, Roggo A, et al. Sex-dependent association between inflammation, neural stress responses, and impaired myocardial function. Eur J Nucl Med Mol Imaging 2019.

19. Oikonomou EK, Marwan M, Desai MY, Mancio J, Alashi A, Hutt Centeno $\mathrm{E}$, et al. Non-invasive detection of coronary inflammation using computed tomography and prediction of residual cardiovascular risk (the CRISP CT study): A post-hoc analysis of prospective outcome data. Lancet 2018;392:929-39.

20. Fuchs TA, Stehli J, Bull S, Dougoud S, Clerc OF, Herzog BA, et al. Coronary computed tomography angiography with modelbased iterative reconstruction using a radiation exposure similar to chest X-ray examination. Eur Heart J 2014;35:1131-6.

Publisher's Note Springer Nature remains neutral with regard to jurisdictional claims in published maps and institutional affiliations. 